\title{
UMA FERRAMENTA PARA AVALIAÇÃO DE RESULTADOS DE DIVERSOS MODELOS DE OTIMIZAÇÃO DE DIETAS
}

\author{
Anderson Amendoeira Namen \\ Cláudio Thomás Bornstein * \\ Engenharia de Sistemas e Computação / COPPE \\ Universidade Federal do Rio de Janeiro (UFRJ) \\ Rio de Janeiro - RJ \\ ctbornst@cos.ufrj.br
}

* Corresponding author/autor para quem as correspondências devem ser encaminhadas

Recebido em 05/2004; aceito em 10/2004 após 1 revisão

Received May 2004; accepted October 2004 after one revision

\section{Resumo}

O presente trabalho visa apresentar uma ferramenta para avaliação de modelos matemáticos relacionados à otimização do planejamento de dietas. Inicialmente, o problema original da dieta é caracterizado, bem como sua importância para o desenvolvimento da Pesquisa Operacional, seus desdobramentos e aplicações posteriores. Em seguida, são apresentados os diversos enfoques e técnicas adotadas para a elaboração de dietas para seres humanos. Alguns desses modelos são então implementados, efetuando-se uma análise comparativa dos resultados obtidos. Para isso, é utilizado um software que permite a definição de parâmetros relacionados às demandas humanas, restrições adicionais relacionadas às quantidades de alimentos e escolha de alguns dos modelos apresentados. Finalmente, o estudo é concluído constatando-se a utilidade e a aplicação da ferramenta não somente para o planejamento de dietas, mas também para a área de ensino de Pesquisa Operacional.

Palavras-chave: problema da dieta; programação linear; otimização.

\begin{abstract}
This paper presents an interactive tool which allows the comparison of different mathematical models for the menu planning problem. First, the original diet problem is characterized, as well as its importance to the development of operational research, its evolution and later applications. Several models for the diet problem are presented. Models are run and results are compared. This is done using an interactive tool which allows the definition of factors related to human needs, additional restrictions related to the amount of food and the selection of appropriate models. This tool may also be used for teaching purposes.
\end{abstract}

Keywords: diet problem; linear programming; optimization. 


\section{Introdução e Histórico}

Em 1945, George Stigler apresentou o seguinte problema (Stigler, 1945): para um homem mediano pesando aproximadamente $70 \mathrm{~kg}$, qual quantidade dentre 77 diferentes alimentos deveria ser ingerida diariamente, de modo que as necessidades mínimas de nutrientes fossem iguais às recomendadas pelo Conselho Nacional de Pesquisa Norte-americano e, além disso, a dieta elaborada tivesse o menor custo possível.

Nessa época, o método simplex ainda não havia sido proposto por George Dantzig, e Stigler resolveu um conjunto amplo de inequações $(9$ x 77) através de uma heurística inteligente, obtendo um custo total para a dieta de 39,93 dólares por ano. Nesse processo, foram examinadas manualmente 510 diferentes possibilidades de combinação de alimentos. Apesar do valor não poder ser considerado exatamente o custo mínimo, devido à inexistência de processo matemático que comprovasse o fato, Dantzig afirma que Stigler apresentou diversas justificativas indicando que o valor encontrado por ele estaria muito próximo do custo mínimo exato (Lustig, 2001).

Posteriormente, em 1947, Dantzig apresentou o método até hoje conhecido e largamente utilizado denominado simplex. Após apresentá-lo, Dantzig necessitava encontrar um bom problema para testar o novo método criado, sendo o problema da dieta de Stigler escolhido para isso. No outono de 1947, Jack Laderman, responsável pelo Projeto de Tabelas Matemáticas do Bureau Nacional de Padrões norte-americano, decidiu então resolver o primeiro problema de "computação" de grande escala com o método simplex de Dantzig. Com o apoio de nove pessoas utilizando calculadoras de mesa de operação manual, após a utilização de um recurso estimado de 120 dias-homem, obteve-se o custo final da dieta de Stigler a 39,69 dólares (Dantzig, 1990) - apenas 24 centavos de dólar menos que o valor originalmente obtido por Stigler.

Mas a narrativa dos primeiros trabalhos de Dantzig relacionados à Programação Linear não termina aqui. Em um depoimento muito bem-humorado, Dantzig (1990) narra a passagem em que, por conta própria, decide utilizar o problema da dieta para redução de seu próprio peso. As diversas simulações do problema, utilizando um computador IBM 701 pertencente à RAND Corporation, que incluíam resultados esdrúxulos (como por exemplo, a sugestão de uma refeição contendo 500 galões de vinagre), foram a base para o nascimento das restrições indicando limites máximos para as variáveis utilizadas no modelo.

Além de Dantzig, muitos outros pesquisadores, ao longo do tempo, fizeram referência ao problema original, incorporando novos alimentos, utilizando novas especificações de nutrientes ou de necessidades humanas mínimas. Smith (1963) apresenta alguns desses trabalhos caracterizando-os como "modelos puramente nutricionais", que buscavam o objetivo final semelhante ao de Stigler: dieta com menor custo que atendesse às recomendações mínimas de nutrientes. Na área militar, particularmente na Rand Corporation, foram publicados artigos como Kao (1962) e até mesmo simulações das possíveis dietas a serem empregadas aos sobreviventes de um ataque nuclear aos Estados Unidos (Pogrund, 1966). Em todos esses trabalhos, a grande dificuldade encontrada era a obtenção de dietas palatáveis. As deficiências encontradas nesses modelos levaram alguns investigadores a criar novos modelos - apresentados no presente trabalho - visando à melhoria da palatabilidade. Mais recentemente, Garille \& Gass (2001) revisitam o problema, atualizando o conjunto de alimentos, preços, e obtendo novos valores para o custo otimizado. 
Modelos relacionados à otimização de dietas passam a ser utilizados também em escolas e Universidades. Segundo Garille \& Gass (2001), o problema da dieta de Stigler e suas modificações são ideais para a sala de aula. Entre estes trabalhos podemos destacar a seleção de um menu do McDonald's (Bosh, 1993) e também os artigos de Erkut (1994) e Liberatore \& Nydick (1999). Outro trabalho (Czyzyk et al., 1999) permite que estudantes tentem, por conta própria, criar dietas que satisfaçam aos requerimentos nutricionais especificados de acordo com o RDA (Recommended Dietary Allowances - Conselho Nacional de Pesquisa Americano). Além do caráter pedagógico, modelos matemáticos de dietas formam a base para programas do Governo norte-americano, como o Planejamento Econômico Alimentar, originalmente desenvolvido pelo Serviço de Pesquisa Agrícola (Agricultural Research Service, 1975).

$\mathrm{O}$ problema da dieta não fica restrito apenas aos humanos. Facilmente ele passa a se adequar à formulação de rações para animais. Nesse caso, a palatabilidade não é mais um fator essencial. Diversas propostas surgem: a geração de rações para o gado de modo interativo através do uso de uma simples calculadora TI59 (France, 1982); a utilização da Programação Linear Paramétrica para determinação do custo mínimo da ração de gado (Glen, 1980); um método para determinação da política ótima de alimentação de porcos utilizando Programação Dinâmica (Glen, 1983). A elaboração de rações passa a ser um dos aspectos relacionados ao retorno financeiro de uma fazenda, integrando-a às colheitas e a alternativas como a construção de unidades de armazenamento (Lazarus \& Kirkman, 1980). Além da produção de rações, a otimização da composição de fertilizantes, que contenham os nutrientes necessários além de um custo mínimo, tem como base o problema da dieta. Lyons \& Dodd (1976) apresentam um método para minimizar os custos totais de fertilizantes a partir do aproveitamento do estrume de animais em conjunto com fertilizantes químicos.

A partir da evolução apresentada, fica claro a importância do problema original proposto por Stigler para o desenvolvimento da Programação Linear, bem como seus desdobramentos e aplicações posteriores. Mais especificamente, analisando o cenário nacional e a questão alimentar, abre-se a oportunidade da aplicação de diferentes modelos matemáticos visando à melhoria da alimentação da população brasileira, em particular as diversas comunidades carentes do nosso país. Determinadas ações do Governo, como o Programa Fome Zero, podem ser amparadas por aplicações na área de otimização relacionadas à elaboração de dietas, que atendam às particularidades locais - cultura regional, características próprias dos indivíduos atendidos, preferências alimentares - e sejam caracterizadas pelo atendimento aos requerimentos nutricionais conjugados à minimização dos custos envolvidos.

Dentro dessa perspectiva, na seção dois são apresentados diversos modelos matemáticos adotados para a elaboração de dietas. Em seguida, na seção três, é apresentada a ferramenta desenvolvida para a avaliação dos resultados de alguns desses modelos. Na seção quatro, os diferentes resultados obtidos são analisados e comparados. Finalmente, a seção cinco apresenta algumas conclusões relacionadas ao presente artigo.

\section{Apresentação de Diversos Modelos Matemáticos}

O problema original proposto por Stigler (1945), apesar de clássico e extremamente importante na área de Pesquisa Operacional, não produzia dietas aceitáveis para consumo. Em sua formulação, o objetivo final era gerar uma dieta com menor custo, que atendesse às restrições mínimas de nutrientes, a partir do seguinte modelo: 
Minimize $\sum_{j=1}^{J} c_{j} x_{j}$

sujeito a

$$
\sum_{j=1}^{J} n_{i j} x_{j} \geq b_{i} \quad \text { para } i=1,2, \ldots, I
$$

$x_{j} \geq 0$ para todo $j$

onde

$\{1,2, \ldots, J\}=$ conjunto de índices dos alimentos disponíveis para a formulação da dieta

$c_{j}=$ custo unitário do j-ésimo alimento

$x_{j}=$ quantidade do j-ésimo alimento presente na dieta

$\{1,2, \ldots, I\}=$ conjunto de índices dos nutrientes

$n_{i j}=$ quantidade do i-ésimo nutriente em uma unidade do j-ésimo alimento

$b_{i}=$ quantidade mínima necessária do i-ésimo nutriente

Smith (1963) apresenta uma pequena variação a essa abordagem. Relações de proporcionalidade entre os alimentos são incluídas no modelo. Para isso, uma série de restrições são incorporadas pelo usuário, de modo que as diferentes relações entre alimentos produzam uma dieta palatável. Para facilitar o entendimento, poderíamos imaginar uma salada contendo aipo, maçã, salsa e maionese. Considerando que deveríamos ter entre $100 \mathrm{~g}$ e $200 \mathrm{~g}$ de salada e que esta deveria ser composta de 4 a 6 por cento de maionese, 4 a 6 por cento de salsa, 30 a 40 por cento de maçã e 50 a 60 por cento de aipo, as seguintes restrições adicionais seriam incluídas no modelo de Stigler:

$$
\begin{aligned}
& x_{1}+x_{2}+x_{3}+x_{4}=y \\
& 0,04 y \leq x_{1} \leq 0,06 y \\
& 0,04 y \leq x_{2} \leq 0,06 y \\
& 0,30 y \leq x_{3} \leq 0,40 y \\
& 0,50 y \leq x_{4} \leq 0,60 y \\
& 100 \leq y \leq 200
\end{aligned}
$$

onde

$$
\begin{aligned}
& x_{1}=\text { quantidade de maionese na salada } \\
& x_{2}=\text { quantidade de salsa na salada } \\
& x_{3}=\text { quantidade de maçã na salada } \\
& x_{4}=\text { quantidade de aipo na salada } \\
& y=\text { quantidade de salada }
\end{aligned}
$$

O grande problema dessa proposta é a necessidade de criação de um grande número de restrições, o que pode contribuir para a não viabilidade das soluções. Além disso, é necessária a análise de diversas combinações diferentes de alimentos, tornando-a impraticável (Lancaster, 1992). 
Czyzyk et al. (1999) seguem a linha de abordagem de Smith, mas criam um processo interativo para a criação das restrições. Interagindo com um software, o usuário pode selecionar os alimentos a incluir em sua dieta, a partir de uma lista de itens apresentados. Após essa seleção, o usuário pode impor as suas próprias restrições (por exemplo, não aceitar mais do que duas bananas por dia, ou pelo menos um tomate por dia incluso em sua dieta). $\mathrm{O}$ software, então, efetua os cálculos, gerando a dieta ou apresentando a mensagem de que nenhuma solução foi encontrada. Nesse último caso, o programa desenvolvido por Czyzyk et al. (1999) faz sugestões de alterações possíveis para possibilitar a viabilidade da dieta. No caso da geração de dietas não palatáveis, o processo interativo permite a inclusão de novas restrições até que um resultado satisfatório seja encontrado. Outras propostas de interatividade são apresentadas por Bosh (1993) e Erkut (1994). Apesar de estarem restritos a alimentos oferecidos pelo McDonald's, os trabalhos indicam a possibilidade de criação de dietas palatáveis dentro de parâmetros limitados de custos, através de uma abordagem interativa. Erkut apresenta, inclusive, a possibilidade interessante de utilizar o EXCEL como ferramenta de fácil entendimento para a resolução dos modelos.

Anderson \& Earle (1983), por sua vez, apresentam uma proposta de elaboração de dietas a partir da minimização dos desvios de nutrientes. $\mathrm{O}$ artigo não discute o problema da palatabilidade, mas sim a necessidade de um melhor balanceamento dos nutrientes. Ele surge a partir da constatação de que o modelo de Stigler gera resultados que apresentam uma quantidade de nutrientes que supera para alguns casos os valores mínimos exigidos. Uma solução seria especificar também limites máximos para estes nutrientes (cabe ressaltar que o modelo de Stigler usa somente restrições que estabelecem níveis mínimos). Isso pode, no entanto, levar à incorporação de uma grande quantidade de restriç̃os adicionais, correndo-se o risco de tornar a solução impossível. Face a esta impossibilidade da solução, a alternativa seria relaxar progressivamente as restrições introduzidas, o que no entanto pode ser muito trabalhoso. A proposta então apresentada por Anderson e Earle, é a Programação por Objetivos (Goal Programming), uma extensão da Programação Linear, que permite a consideração de diversos objetivos simultaneamente. A função objetivo considerada no trabalho é:

$$
\begin{aligned}
& \text { Minimize } \sum_{j=1}^{J} a_{i}\left(n_{i j} x_{j}-b_{i}\right) \\
& \text { sujeito a } \\
& \sum_{j=1}^{J} n_{i j} x_{j} \geq b_{i} \text { para } i=1,2, \ldots, I \\
& x_{j} \geq 0 \text { para todo } j \\
& \text { onde } \\
& a_{i}=\text { peso relacionado ao nutriente } i
\end{aligned}
$$

O objetivo passa a ser minimizar o desvio total dos níveis de nutrientes obtidos em relação aos requerimentos especificados nas restrições do modelo. Anderson e Earle aplicam a Programação por Objetivos, encontrando um custo aproximadamente 3 vezes maior em comparação com o modelo de Stigler, concluindo-se que uma nova restrição poderia ser colocada no modelo, de modo que os custos totais fossem limitados a um valor máximo:

$$
\sum_{j=1}^{J} c_{j} x_{j} \leq C \text { onde } C=\text { custo máximo e } c_{j}=\text { custo unitário do } j \text {-ésimo alimento }
$$


Desse modo, seria possível chegar a uma combinação satisfatória entre os fatores custo e balanceamento nutritivo. Apesar disso, fica claro que uma grande deficiência do modelo é o tratamento insatisfatório da questão do custo bem como a incerteza acerca da palatabilidade da dieta.

Entre as evoluções em relação ao modelo original, podemos citar a utilização de variáveis simbolizando receitas já preparadas, ao invés de variáveis representando alimentos. Por exemplo, em vez de utilizar a cenoura, poderia ser utilizado o suflê de cenoura, que englobaria mais de um ingrediente e que teria, por sua vez, os respectivos valores nutritivos considerados nas equações do modelo. Além disso, o planejamento das dietas pode considerar um conjunto de dietas vinculado a períodos de tempo, com equações relacionadas à preferência do consumidor variando ao longo do tempo.

Um primeiro modelo (Balintfy, 1975) foi definido utilizando quantidades padrão prédefinidas das receitas como variáveis, e objetivava minimizar o custo a partir de restrições relacionadas à composição das refeições e à repetição de receitas ao longo do tempo. Para isso, Balintfy trabalhou com $K$ diferentes categorias de receitas, não permitindo que duas diferentes receitas de uma mesma categoria pudessem pertencer à mesma refeição. Para facilitar a compreensão do problema, poderíamos ilustrar a categoria carnes, como composta das seguintes receitas: bife de fígado, filé de pescado, frango ensopado, carne (lagarto) assada, bife de contra-filé. Assim, essas diferentes receitas não poderiam estar presentes, juntas, na mesma refeição. Além disso, a mesma receita não poderia ser repetida na elaboração de uma refeição ao longo de um período de tempo $t$. O conjunto de equações a seguir representa este modelo, sendo que o mesmo é resolvido iterativamente para cada tempo $t$ e o valor do custo global é a soma dos valores obtidos em todas as iterações:

$$
\begin{array}{ll}
\text { Minimize } \sum_{k=1}^{K} \sum_{j \in N_{k}} c_{k j} x_{k j}(t) & \\
\text { sujeito a } & \\
\sum_{k=1}^{K} \sum_{j \in N_{k}} a_{i k j} x_{k j}(t) \geq b_{i} & \text { para } i=1,2, \ldots, I \\
\sum_{j \in N_{k}} x_{k j}(t)=1 & \text { para } k=1,2, \ldots, K \\
x_{k j}(t)=0 \text { ou } 1 & \text { para } j \in N_{k}, k=1,2, \ldots, K \\
x_{k j}(t)=0 \quad \text { se } s_{k j}(t)>0 & \text { para } j \in N_{k}, k=1,2, \ldots, K \\
s_{k j}(1)=0 & \text { para } j \in N_{k}, k=1,2, \ldots, K \\
s_{k j}(t+1)=\max _{k j}\left(s_{k j}(t)-1,0\right] & \text { se } x_{k j}(t)=0, \text { para } t \geq 1, j \in N_{k}, k=1,2, \ldots, K \\
s_{k j}(t+1)=s_{k j}^{\prime} & \text { se } x_{k j}(t)=1, \text { para } t \geq 1, j \in N_{k}, k=1,2, \ldots, K
\end{array}
$$

onde

$\{1,2, \ldots, I\}=$ conjunto de índices dos nutrientes;

$\{1,2, \ldots, K\}=$ conjunto de índices das categorias de receitas;

$b_{i}=$ quantidade do i-ésimo nutriente;

$c_{k j}=$ custo da j-ésima receita pertencente à k-ésima categoria de receitas;

$a_{i k j}=$ quantidade do i-ésimo nutriente na j-ésima receita pertencente à k-ésima categoria; 
$s^{\prime}{ }_{k j}=$ intervalo de utilização da j-ésima receita da k-ésima categoria, sempre inferior ao número total de receitas relacionadas à categoria;

$N_{k}=$ conjunto de receitas pertencentes à categoria $\mathrm{k}$;

$x_{k j}(t)=$ indicador se a porção padrão da j-ésima receita pertencente à categoria $\mathrm{k}$ faz parte ou não da dieta no tempo $t$;

$t=$ escala de tempo relacionada a períodos entre refeições iniciando em $t=1$.

É importante ressaltar que $s_{k j}(t)$ é tão somente uma variável auxiliar que visa garantir um intervalo de tempo mínimo para a repetição da mesma receita. Assim, as equações (13) a (16) indicam que cada receita $j$ não será repetida durante, no mínimo, o intervalo de tempo igual a $s_{k j}^{\prime}$. Por exemplo, sejam $x_{11}(t), x_{12}(t), x_{13}(t)$ as variáveis representando, respectivamente, a presença de uma porção de bife de fígado, filé de pescado e frango ensopado, na dieta. Se tivéssemos $s_{11}^{\prime}=1, s_{12}^{\prime}=2$ e $s_{13}^{\prime}=3$, isto significaria que o bife de fígado poderia entrar na dieta a cada dois dias, o filé de pescado só poderia estar na dieta de três em três dias, e o frango ensopado somente faria parte da dieta em intervalos de tempo superiores ou iguais a quatro.

Conforme já mencionado, os resultados gerados pelo modelo de Balintfy relacionam-se a quantidades pré-definidas das receitas. Por exemplo, se uma receita de suflê de cenoura é pré-definida com a quantidade de $100 \mathrm{~g}$, a dieta obtida possui $100 \mathrm{~g}$ ou $0 \mathrm{~g}$ de suflê de cenoura. Isso implica em grande perda de liberdade e do potencial de produzir soluções economicamente vantajosas. Armstrong \& Sinha (1974) desenvolvem um modelo semelhante, contudo sua abordagem permite que cada variável $x_{k j}(t)$ possa conter o valor zero ou então $1-\varepsilon \leq x_{k j}(t) \leq 1+\varepsilon$. Isto leva a variáveis próximas do valor inteiro, o que pode explicar a expressão "variáveis quase-inteiras", utilizada por Bénichou et al. (1971). Ao invés de apresentar porções fixas de cada receita (ex.: $100 \mathrm{~g}$ de bife de fígado, $200 \mathrm{~g}$ de frango, etc), o tamanho dessas porções pode variar em um pequeno percentual, até porque pequenas variações são viáveis operacionalmente. A resolução do modelo com uma variação permitida de no máximo $10 \%$ nas porções das receitas, levou a um custo aproximadamente $8,5 \%$ inferior ao do modelo de Balintfy (Armstrong \& Sinha, 1974). O modelo que representa o exposto é:

Minimize $\sum_{k=1}^{K} \sum_{j \in N_{k}} c_{k j} x_{k j}(t)$

sujeito a

$$
\begin{array}{ll}
\sum_{k=1}^{K} \sum_{j \in N_{k}} a_{i k j} x_{k j}(t) \geq b_{i} & \text { para } i=1,2, \ldots, I \\
x_{k j}(t)=0 \text { ou } v_{k j} \leq x_{k j}(t) \leq u_{k j} & \text { para } j \in N_{k}, k=1,2, \ldots, K \\
\exists j \in N_{k} \mid x_{k j} \geq v_{k j} & \text { para } k=1,2, \ldots, K \\
\forall j, l \in N_{k}, \quad j \neq l \quad \text { temos } & \left(v_{k j} \leq x_{k j}(t) \leq u_{k j}\right) \Rightarrow x_{k l}(t)=0 \\
x_{k j}(t)=0 \quad \text { se } s_{k j}(t)>0 & \text { para } j \in N_{k}, k=1,2, \ldots, K \\
s_{k j}(1)=0 & \text { para } j \in N_{k}, k=1,2, \ldots, K \\
s_{k j}(t+1)=\max \left[s_{k j}(t)-1,0\right] & \text { se } x_{k j}(t)=0, \text { para } t \geq 1, j \in N_{k}, k=1,2, \ldots, K \\
s_{k j}(t+1)=s_{k j}^{\prime} & \text { se } x_{k j}(t)=1, \text { para } t \geq 1, j \in N_{k}, k=1,2, \ldots, K
\end{array}
$$


A equação (19) substitui (12) e as equações (20) e (21) substituem (11). Podemos observar que $x_{k j}$ é uma variável quase-inteira e que, se fizermos $v_{k j}=u_{k j}=1$, a variável passa a ser inteira e retornamos ao modelo de Balintfy. Além disso, as restrições (19) a (25) poderiam ser representadas matematicamente, a partir da utilização de uma variável auxiliar $y_{k j}$, na seguinte forma:

$$
\begin{array}{ll}
\sum_{j \in N_{k}} y_{k j}(t)=1 & \text { para } k=1,2, \ldots, K \\
y_{k j}(t)=0 \text { ou } 1 & \text { para } j \in N_{k}, k=1,2, \ldots, K \\
y_{k j}(t)=0 \quad \text { se } s_{k j}(t)>0 & \\
s_{k j}(1)=0 & \text { para } j \in N_{k}, k=1,2, \ldots, K \\
s_{k j}(t+1)=\max \left[s_{k j}(t)-1,0\right] & \text { se } y_{k j}(t)=0, \text { para } t \geq 1, j \in N_{k}, k=1,2, \ldots, K \\
s_{k j}(t+1)=s^{\prime}{ }_{k j} & \text { se } y_{k j}(t)=1, \text { para } t \geq 1, j \in N_{k}, k=1,2, \ldots, K \\
x_{k j}(t) \geq(1-\varepsilon) y_{k j}(t) & \\
x_{k j}(t) \leq(1+\varepsilon) y_{k j}(t) &
\end{array}
$$

Verifica-se, pelas equações acima, que $(1-\varepsilon) y_{k j}(t)$ substitui $v_{k j}$ e $(1+\varepsilon) y_{k j}(t)$ substitui $u_{k j}$. A variação máxima relacionada às quantidades fixas das receitas é representada por $\varepsilon$. Por exemplo, se a variação máxima desejada nas porções das receitas fosse de 10 por cento, $\varepsilon$ seria igual a 0,1 .

As experiências com a elaboração de menus a partir dos modelos apresentados levaram à uma nova geração de modelos de planejamento de dietas, caracterizada pela quantificação das preferências de consumo. Dentro dessa perspectiva, alguns trabalhos (Balintfy et al., 1974 e Balintfy et al., 1978) especificam algumas funções que representam as preferências alimentares ao longo do tempo, de acordo com a freqüência de consumo de determinado alimento. Com base nessas funções, Balintfy (1979) calcula o custo de uma dieta que atende às necessidades de alimentação criando um modelo onde a função objetivo não é mais a minimização do custo. Nesse novo modelo, passa-se a maximizar uma função que exprime a preferência alimentar como função da freqüência de consumo do alimento, com o custo máximo passando a fazer parte do conjunto de restrições. Mais recentemente, Balintfy et al. (1996) incluem no seu modelo a variação dos preços dos alimentos ao longo do tempo.

Em todas as propostas apresentadas até aqui, a definição das refeições é feita automaticamente pelo computador, com exceção das propostas de Czyzyk et al. (1999), Bosh (1993) e Erkut (1994), que permitem ao usuário interagir com o sistema para a escolha dos alimentos. Esses trabalhos, contudo, são protótipos voltados à educação, objetivando incentivar os estudantes no entendimento de alguns métodos da área de Pesquisa Operacional.

Lancaster (1992), por sua vez, apresenta um modelo para elaboração seletiva de refeições, através da interatividade com o planejador das dietas. Segundo Lancaster (1992), a definição interativa de refeições é preferida, devido à heterogeneidade das populações e ao valor dado pelos consumidores à liberdade de escolha. Em sua abordagem, o especialista em planejamento de refeições define uma combinação inicial de receitas com porções padronizadas. Esses itens são escolhidos com base em uma lista previamente preparada definida pelo próprio especialista - que considera aspectos de compatibilidade entre 
diferentes alimentos. Segundo Lancaster (1992), muitas refeições elaboradas, num primeiro momento, não atendem aos objetivos nutricionais. Por isso é necessário ajustar os tamanhos das porções, de modo a atender a esses requerimentos. Lancaster (1987) desenvolve uma aplicação que permite a interação entre computador e especialista, de modo que estes requerimentos sejam atingidos.

\section{Geração de Modelos}

Com o objetivo de facilitar o processo de elaboração de dietas otimizadas a partir de alguns dos diferentes enfoques apresentados anteriormente, foi desenvolvido um software compatível com o sistema operacional Windows que possibilitou a análise dos resultados da implementação de diferentes modelos matemáticos. O aplicativo - denominado SimulaDieta - foi desenvolvido utilizando a linguagem Visual Basic for Applications em conjunto com o Gerenciador de Banco de Dados ACCESS. A partir do acesso a uma base de dados em ACCESS, o software gera, em um arquivo, o modelo matemático solicitado pelo usuário, contendo a sintaxe requerida pelo LINDO - programa computacional que soluciona problemas de otimização (Schrage, 1997). Quatro modelos apresentados na seção 2 podem ser avaliados através da ferramenta:

- modelo original de Stigler, equações (1) a (3);

- modelo com minimização de desvios de nutrientes (Anderson \& Earle, 1983), equações (5) a (8);

- modelo de Balintfy (1975), equações (9) a (16);

- modelo de Balintfy com variáveis quase-inteiras (Armstrong \& Sinha, 1974), equações (17) a (25).

O software gera modelos matemáticos que visam à otimização de dietas que atendam às necessidades diárias de uma pessoa. Cabe ressaltar que, apesar de um dos objetivos principais dos modelos de Balintfy (1975) e de Armstrong \& Sinha (1974) ser a não repetição de refeições dentro de um certo intervalo de tempo, a ferramenta desenvolvida, cujos resultados serão apresentados nesta seção, calcula somente uma única dieta. Ou seja, foram rodados os modelos de Balintfy e de Armstrong \& Sinha fazendo-se $t=1$. Evidentemente, temos então $s_{k j}(1)=0$ para todo $k, j$, o que possibilita a utilização de qualquer alimento. Isto foi feito para possibilitar melhor comparação entre os resultados dos diversos modelos, já que os modelos de Stigler e de minimização de desvios de nutrientes determinam uma única dieta. Além disso, conforme será mencionado mais adiante, para facilitar a comparação entre os modelos, utilizamos os modelos de Balintfy e de Armstrong \& Sinha com alimentos crus ao invés de receitas.

Ao utilizar o software, o usuário pode escolher a refeição ou refeições para as quais a dieta será elaborada, bem como o percentual da demanda diária de nutrientes a ser atendido por estas refeições. Por exemplo, o usuário pode decidir gerar uma dieta para o café da manhã que atenda apenas a vinte por cento das necessidades diárias de nutrientes, ou então gerar uma dieta para almoço que atenda a quarenta por cento das demandas necessárias. Cabe lembrar mais uma vez, que a resposta da rodada de cada modelo será uma única dieta que visa atender a uma ou mais refeições (por exemplo, almoço e jantar) diárias, suprindo um certo percentual das necessidades diárias de nutrientes. 
A base de dados utilizada é composta pelas seguintes informações:

- composições de 43 diferentes alimentos no estado cru, disponibilizadas pelo Instituto Brasileiro de Geografia e Estatística (IBGE, 1996). Apesar de alguns dos modelos apresentados (Balintfy, 1975 e Armstrong \& Sinha, 1974) serem caracterizados pela utilização de receitas combinando diversos alimentos, para melhor facilitar a comparação entre os resultados dos diferentes modelos optou-se pela elaboração de dietas contendo somente alimentos crus. Os 43 alimentos utilizados foram escolhidos de modo a possibilitar uma gama razoável de alimentos e atender a características particulares de alimentação da região Sudeste do Brasil, em particular o Estado do Rio de Janeiro;

- tabela com valores diários de nutrientes recomendados de acordo com as diferentes categorias dos indivíduos (Institute of Medicine - Food and Nutrition Board, 2001);

- tabela de preços médios dos alimentos no Brasil no ano de 2002 (IBGE, 2002);

- dados fornecidos pelo usuário, apresentando informações suplementares para cada alimento: categoria a que pertence dentro dos diferentes grupos alimentares definidos pelos nutricionistas (Franco, 2003) e as refeições em que pode ser utilizado (café da manhã, almoço, lanche da tarde, jantar).

A Figura 1 mostra o mapa de navegação entre as janelas do sistema e a função de cada uma delas. Cada quadro retangular representa uma janela. A flecha indica a chamada de uma janela para outra.

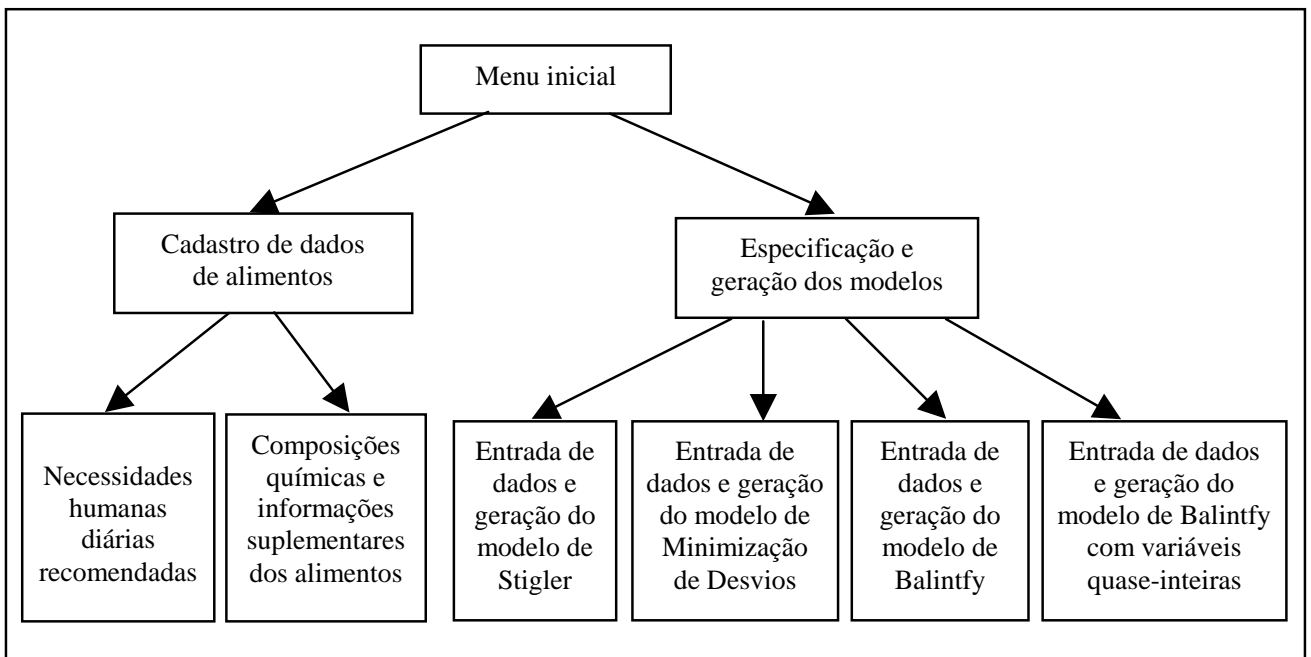

Figura 1 - Esquema de navegação entre as janelas do software SimulaDieta

O software apresenta um menu inicial, onde o usuário pode escolher entre duas diferentes opções: Cadastro de Dados de Alimentos e Especificação e Geração dos Modelos. Escolhendo a primeira opção, será apresentado um novo menu permitindo a escolha de uma entre as seguintes opções de cadastros: Necessidades Humanas Diárias Recomendadas e Composições Químicas e Informações Suplementares dos Alimentos. Esses cadastros são a 
base para a elaboração das dietas. Caso o usuário opte pela Especificação e Geração dos Modelos, será solicitada a entrada dos dados referentes ao perfil da pessoa e dieta e a escolha de um entre quatro diferentes modelos, conforme a Figura 2.

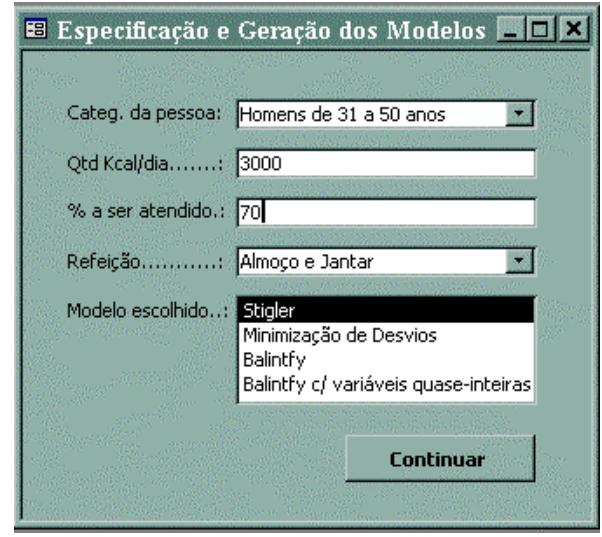

Figura 2 - Formulário para especificação e escolha do modelo a ser gerado

Como pode ser observado, o usuário poderá escolher a categoria da pessoa para a qual será elaborada a dieta (por exemplo: homens de 19 a 30 anos, homens de 31 a 50 anos, mulheres de 19 a 30 anos, etc). A partir dessa escolha, o sistema definirá as quantidades de nutrientes necessárias para a respectiva categoria. O usuário deverá definir também a quantidade diária de calorias necessária para o atendimento das necessidades humanas. Essa quantidade é definida de acordo com a energia demandada para o nível de atividade física diário; por exemplo, homens de 31 a 50 anos com alto nível de atividade física demandarão mais calorias do que homens de 31 a 50 anos com atividade física moderada. Finalmente, podem ser elaboradas dietas que atendam a um determinado percentual em relação às necessidades diárias recomendadas (campo \% a ser atendido) e podem ser escolhidas as refeições que serão consideradas para essa dieta (ex.: café da manhã, almoço, jantar, lanche da tarde). Conforme já apresentado nessa seção, essas opções permitem ao usuário definir dietas que atendam de modo parcial às necessidades diárias humanas. Nas avaliações feitas no presente trabalho e apresentadas na seção 4, foram consideradas apenas as refeições de almoço e jantar, assumindo-se que elas atenderiam a setenta por cento das necessidades diárias de uma pessoa.

A partir da entrada desses dados, o usuário pode gerar um dentre quatro modelos, sendo que cada um dos modelos exige, adicionalmente ao "Cadastro de dados de alimentos", uma entrada específica. Trata-se de especificações adicionais para cada modelo e que consistem em:

- Modelo original de Stigler: entrada de restrições adicionais ao modelo, visando melhorar a palatabilidade das dietas. O modelo de Stigler pode gerar algumas dietas com quantidades excessivas de determinados alimentos; nesse caso, restrições adicionais poderiam ser adicionadas, limitando essas quantidades a um valor máximo;

- Modelo com minimização de desvios de nutrientes: entrada dos nutrientes que terão o seu desvio minimizado e da restrição com o custo máximo permitido;

- Modelo de Balintfy: entrada das quantidades pré-definidas dos alimentos. Como esse modelo utiliza quantidades padrão pré-definidas dos alimentos, essas quantidades devem 
ser definidas pelo usuário antes do modelo ser gerado. Será verificado mais adiante, que é interessante utilizar os resultados obtidos na avaliação do modelo de Stigler para a definição dessas quantidades, visando à construção de um modelo que contenha soluções viáveis;

- Modelo de Balintfy com variáveis quase-inteiras: entrada das quantidades pré-definidas dos alimentos e do percentual máximo permitido para variação das mesmas.

A Figura 3 apresenta os formulários relacionados aos modelos de Stigler e de minimização de desvios de nutrientes:
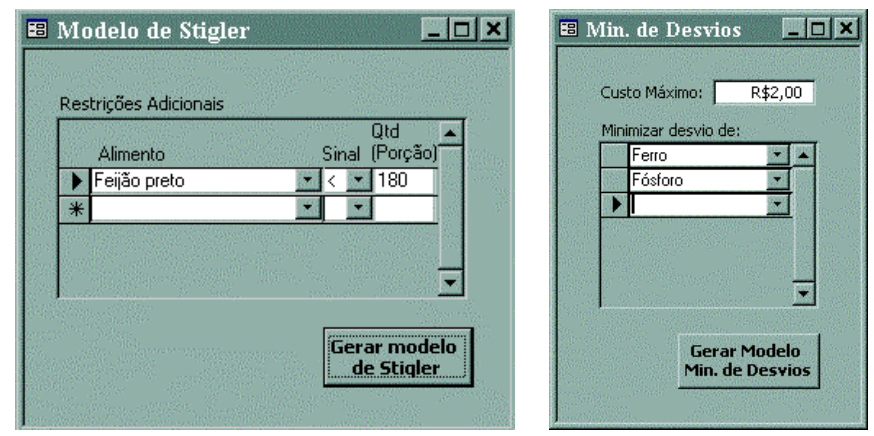

Figura 3 - Formulários para geração dos modelos de Stigler e Minimização de Desvios

Na Figura 4 são apresentados os formulários referentes aos modelos de Balintfy e de Balintfy com variáveis quase-inteiras. Conforme pode ser visto na figura, além da definição das quantidades padrão, é possível que o usuário exclua alguns alimentos do processo de elaboração da dieta, bastando, para isso, desmarcar o campo Usar?.
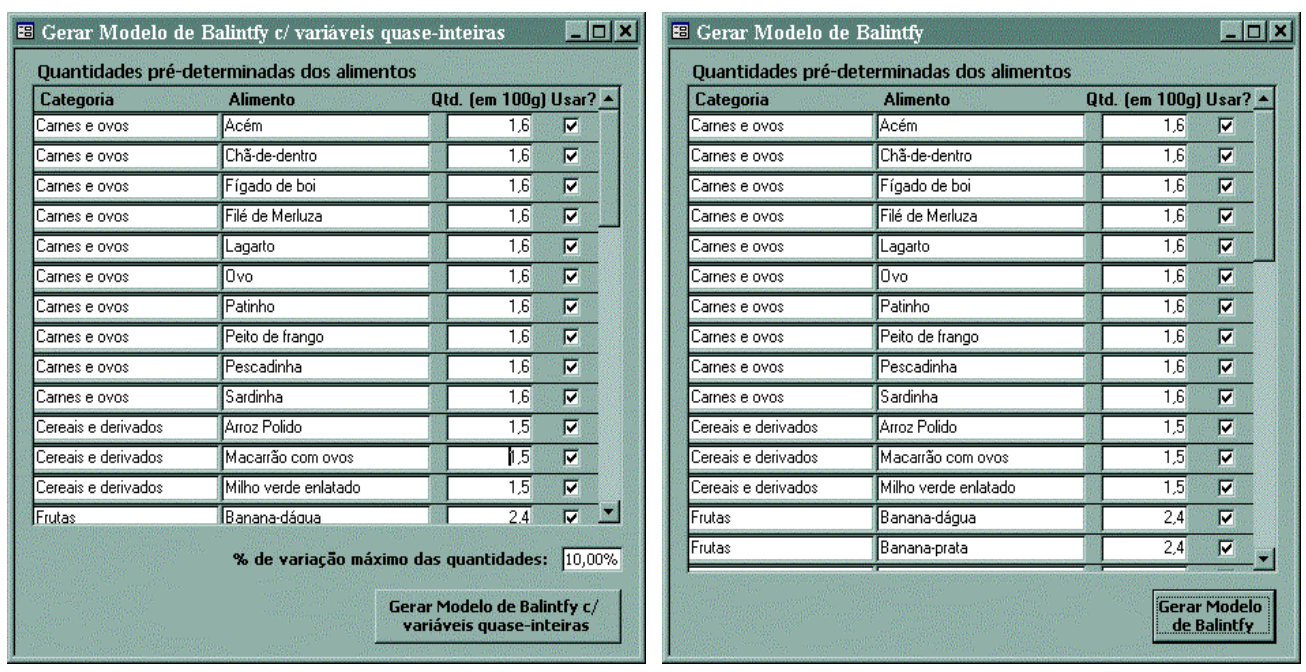

Figura 4 - Formulários para geração dos modelos de Balintfy e Balintfy com variáveis quase-inteiras 


\section{Implementação e Análise de Resultados}

Foram realizadas seis diferentes simulações para a elaboração de dietas diárias utilizando os quatro diferentes modelos, apresentados na Seção 3. Três simulações foram realizadas com o modelo de Stigler introduzindo-se limites para as quantidades de alimentos e as outras três foram realizadas para os modelos de minimização de desvios de nutrientes, Balintfy e Balintfy com variáveis quase-inteiras, respectivamente. De modo a possibilitar uma análise comparativa, todas as simulações utilizaram os seguintes parâmetros:

- categoria de homens de 31 a 50 anos com peso de $65 \mathrm{~kg}$ e atividade física moderada, demandando 3000kcal/dia (Franco, 2003);

- atendimento de $70 \%$ das necessidades mínimas diárias para a categoria acima, considerando a elaboração de dietas para as refeições do almoço e do jantar. Essa escolha foi feita a partir da hipótese de que $70 \%$ das necessidades diárias seriam satisfeitas por essas duas refeições, cabendo os outros $30 \%$ ao café da manhã e outra pequena refeição ao longo do dia. Cabe salientar que essa escolha possibilita o reaproveitamento, no jantar, dos alimentos preparados para o almoço.

Além disso, em todos os modelos avaliados foram agregadas restrições referentes à quantidade máxima permitida dos macro nutrientes - proteínas, lipídios e glicídios - o que, de acordo com Kraus \& Mahan (1985), permite produzir uma dieta mais saudável.

Inicialmente, decidiu-se pela abordagem original de Stigler - equações (1) a (3). O resultado obtido foi um custo de $\mathrm{R} \$ 1,4665 \mathrm{com}$ as seguintes quantidades de alimentos:

- $151,42 \mathrm{~g}$ de arroz

- $183,92 \mathrm{~g}$ de couve

- $268,49 \mathrm{~g}$ de feijão

- $11,98 \mathrm{~g}$ de queijo minas

- $66,7 \mathrm{ml}$ de óleo de soja (ou 61,23g)

Analisando os resultados, podemos concluir sobre a inviabilidade da utilização dessa dieta. A variedade de alimentos é muito pequena e as quantidades de alguns deles muito altas, tornando-a não palatável.

Com o objetivo de melhorar o resultado foram criadas, através da ferramenta, restrições adicionais ao modelo, limitando-se as quantidades máximas de feijão $(110 \mathrm{~g})$, arroz $(160 \mathrm{~g})$ e óleo de soja $(40 \mathrm{~g}=43,5 \mathrm{ml})$. Os parâmetros restantes permaneceram os mesmos. Com isso, foi gerada a seguinte dieta:

- $160 \mathrm{~g}$ de arroz

- $110 \mathrm{~g}$ de feijão

- $2,82 \mathrm{~g}$ de carne de fígado

- $66,05 \mathrm{~g}$ de macarrão

- $1193,66 \mathrm{~g}$ de repolho

- $7,58 \mathrm{~g}$ de queijo minas

- 25,06g de margarina

- 43,5 ml de óleo de soja (ou 40g) 
Podemos verificar o aumento na variedade de alimentos, havendo, no entanto, uma quantidade exagerada de repolho, bem como uma quantidade absurdamente pequena de fígado e de queijo minas. O custo aumenta para $\mathrm{R} \$ 1,7162$, já que as novas restrições implicaram na substituição de alimentos mais baratos por alimentos de maior preço.

A ferramenta permite que o usuário, de forma interativa, defina novas restrições ao modelo, de modo a possibilitar a melhoria das condições de palatabilidade da dieta. Dessa maneira, foi incluída uma nova restrição limitando a quantidade de repolho a um valor máximo de $100 \mathrm{~g}$. Os demais parâmetros e restrições foram mantidos iguais. Foi feita uma nova rodada do modelo tendo sido obtido um custo de $\mathrm{R} \$ 1,7422$ para um almoço e um jantar, constituído das seguintes quantidades de alimentos:

- $160 \mathrm{~g}$ de arroz

- $110 \mathrm{~g}$ de feijão

- $2,55 \mathrm{~g}$ de carne de fígado

- 56,66g de macarrão

- $100 \mathrm{~g}$ de repolho

- $63,17 \mathrm{~g}$ de queijo minas

- $13,45 \mathrm{~g}$ de margarina

- 43,5 ml de óleo de soja (ou 40g)

- $331,23 \mathrm{~g}$ de banana-d'água

- $2,71 \mathrm{~g}$ de sardinha

Novamente notamos o aumento do custo final, além do aumento na variedade de alimentos. Observamos uma melhoria em relação à palatabilidade da dieta, mas constata-se uma grande quantidade de banana-d'água. Nesse sentido, assim como os trabalhos de Czyzyk et al. (1999), Bosh (1993) e Erkut (1994), a ferramenta permite que o usuário ajuste e crie, interativamente, as restrições referentes às quantidades de alimentos, na busca de uma dieta plausível. Fica claro que cada nova restrição adicionada ao modelo implica em aumento do custo. Basta observar o aumento de 18,79 por cento no custo da dieta acima ( $\mathrm{R} \$ 1,7422)$ em relação ao custo da primeira dieta gerada $(\mathrm{R} \$ 1,4665)$, que utilizava o modelo de Stigler sem quaisquer restrições adicionais.

Retornando agora aos resultados da primeira simulação - ou seja, modelo de Stigler sem restrições adicionais - podemos observar que a dieta obtida contém um excedente muito alto de determinados nutrientes. Isso se deve ao fato que esse modelo somente considera quantidades mínimas nas restrições referentes aos micronutrientes (Cálcio, Fósforo, Ferro, Niacina, Vitaminas A, B1, B2 e C). Mais especificamente, na primeira simulação, verifica-se que os nutrientes onde houve maior desvio com relação aos valores especificados foram fósforo, ferro, vitamina A e vitamina C. Quanto ao fósforo, a solução resultou em um excedente de 498,07 mg, o que significa que a quantidade de fósforo contida na dieta foi mais de 100 por cento superior ao mínimo recomendado (490 mg). Quanto ao ferro, o excedente foi de $18,66 \mathrm{mg}$, significando uma quantidade mais de 3 vezes superior ao mínimo recomendado $(5,6 \mathrm{mg})$. Finalmente, a dieta apresentou um excedente de 603,26 mg de vitamina A - indicando que o resultado possuía quase o dobro da quantidade mínima recomendada $(630 \mathrm{mg}$ ) - e de 114,26 $\mathrm{mg}$ de vitamina $\mathrm{C}$, implicando em uma quantidade aproximadamente três vezes superior ao mínimo recomendado $(63 \mathrm{mg})$. 
Com base nesses dados, optou-se por uma nova simulação utilizando-se o modelo de minimização de desvios de nutrientes - equações (5) a (8) - onde a função objetivo passou a ser minimizar os desvios de fósforo, ferro, vitaminas A e C. Além disso, foi incluída uma nova restrição relacionada ao custo total da dieta, limitando-o ao máximo de $\mathrm{R} \$ 2,00$. Os resultados obtidos foram:

- $306,75 \mathrm{~g}$ de arroz

- $61,37 \mathrm{~g}$ de couve

- $0,7 \mathrm{~g}$ de carne de fígado

- $5,85 \mathrm{~g}$ do peixe merluza

- $8,68 \mathrm{~g}$ do peixe sardinha

- $105 \mathrm{~g}$ de maçã

- $62,12 \mathrm{~g}$ de macarrão

- 49,43 ml de óleo de soja (ou 45,38g)

- $60 \mathrm{~g}$ de queijo prato

$\mathrm{O}$ custo encontrado foi igual ao limite máximo da restrição, ou seja, $\mathrm{R} \$ 2,00$. De acordo com Anderson \& Earle (1983), o grande objetivo dessa abordagem é permitir a geração de uma dieta contendo um melhor balanceamento de nutrientes. Isso realmente acontece, já que os excedentes de fósforo, ferro, vitamina A e C são reduzidos de forma considerável, conforme a Tabela 1.

Tabela 1 - Comparação entre os excedentes de nutrientes

\begin{tabular}{|l|c|c|c|c|}
\hline & $\begin{array}{c}\text { Excedente de } \\
\text { Fósforo }\end{array}$ & $\begin{array}{c}\text { Excedente de } \\
\text { Ferro }\end{array}$ & $\begin{array}{c}\text { Excedente de } \\
\text { Vitamina A }\end{array}$ & $\begin{array}{c}\text { Excedente de } \\
\text { Vitamina C }\end{array}$ \\
\hline Modelo de Stigler & $498,07 \mathrm{mg}$ & $18,66 \mathrm{mg}$ & $603,26 \mathrm{mg}$ & $114,26 \mathrm{mg}$ \\
\hline Modelo com Min. de Desvios & $396,31 \mathrm{mg}$ & $1,79 \mathrm{mg}$ & $0 \mathrm{mg}$ & $0 \mathrm{mg}$ \\
\hline
\end{tabular}

Contudo, a dieta dificilmente pode ser considerada viável, pois as quantidades de determinados alimentos, como os peixes e a carne de fígado, são muito pequenas para serem consideradas em uma refeição. Por outro lado, podemos observar que, à medida em que melhoramos o balanceamento nutritivo, um maior número de alimentos passa a ser incorporado na dieta. Ou seja, um melhor balanceamento nutritivo implica na diversidade maior dos alimentos e também na melhoria da palatabilidade. Pensando nisso, passaremos para a simulação do modelo de Balintfy - equações (9) a (16) -, que permite essa diversidade, já que obriga que alimentos pertencentes a diferentes categorias sejam incorporados à dieta.

A simulação utilizando o modelo de Balintfy não considerou receitas prontas, mas sim alimentos crus. Além disso, foi elaborada uma única dieta (ou seja, não houve criação de diversas dietas ao longo do tempo). Todas essas considerações foram feitas com o objetivo de facilitar a comparação entre os resultados dos diferentes modelos. Finalmente, todos os parâmetros apresentados anteriormente foram mantidos e nenhuma restrição adicional relacionada à quantidade dos alimentos foi incluída no modelo.

Relembrando o modelo, cabe mencionar que porções fixas de alimentos são utilizadas, não sendo permitido que alimentos pertencentes ao mesmo grupo estejam presentes na mesma 
dieta. Essa limitação implica na redução do espaço de soluções possíveis. De fato, inicialmente, a simulação gerou modelos sem solução viável (vazios), já que as porções, ou quantidades fixas de alimentos, definidas pelo usuário, não permitiam o atendimento às diversas restrições. Foram necessárias diversas mudanças das quantidades das porções, até que se encontrasse uma solução viável. Essa solução apresentou um custo de R \$2,4200 e foi composta pelos seguintes alimentos:

- $160 \mathrm{~g}$ de arroz (grupo de cereais e derivados)

- $110 \mathrm{~g}$ de feijão (grupo de leguminosas)

- $70 \mathrm{~g}$ de couve (grupo de vegetais do grupo A)

- $70 \mathrm{~g}$ de beterraba (grupo de vegetais do grupo B)

- $130 \mathrm{~g}$ de sardinha (grupo de carnes em geral)

- $190 \mathrm{~g}$ de banana-prata (grupo de frutas)

- $110 \mathrm{~g}$ de pão doce (grupo de pães e similares - poderia ser utilizado na sobremesa)

- $270 \mathrm{~g}$ de queijo prato (grupo de leites e derivados)

- 43,5 ml de óleo de soja (ou 40g) (grupo de óleos e gorduras)

O resultado obtido parece bastante palatável, mas fica evidente a necessidade de um especialista em nutrição interagindo com o software, de modo a facilitar a definição das porções dos diferentes alimentos.

A última simulação efetuada utilizou o modelo de Balintfy com variáveis quase-inteiras equações (17) a (25) - utilizando os mesmos parâmetros, restrições e porções de alimentos do último modelo. Foi permitida uma variação de $10 \%$ nas quantidades dos alimentos, obtendo-se o seguinte resultado:

- $176 \mathrm{~g}(110 \%$ de $160 \mathrm{~g})$ de arroz

- $106,24 \mathrm{~g}(96,59 \%$ de $110 \mathrm{~g})$ de feijão

- $63,76 \mathrm{~g}(91,09 \%$ de $70 \mathrm{~g})$ de couve

- $63 \mathrm{~g}(90 \%$ de $70 \mathrm{~g})$ de beterraba

- $117 \mathrm{~g}(90 \%$ de $130 \mathrm{~g})$ de sardinha

- $171 \mathrm{~g}(90 \%$ de $190 \mathrm{~g})$ de banana-d'água que substituiu a banana-prata no grupo frutas

- $99 \mathrm{~g}(90 \%$ de $110 \mathrm{~g})$ de pão doce

- $243 \mathrm{~g}(90 \%$ de $270 \mathrm{~g})$ de queijo prato

- 47,85 ml (110\% de 43,5ml) de óleo de soja (ou 44g)

A possibilidade de variação nas porções de alimentos aumentou a flexibilidade, permitindo a melhoria dos resultados da função objetivo, com a obtenção de um custo de $\mathrm{R} \$ 2,2306$, $7,82 \%$ inferior ao obtido anteriormente.

\section{Conclusões}

O presente trabalho apresentou uma ferramenta que permite a avaliação de diferentes modelos relacionados à elaboração de dietas humanas. Analisando os resultados obtidos, ficou evidente a relação existente entre o tratamento da palatabilidade e a redução do espaço de soluções viáveis, implicando no aumento do custo da dieta. A comparação dos diferentes modelos pode ser ilustrada pela Figura 5. 


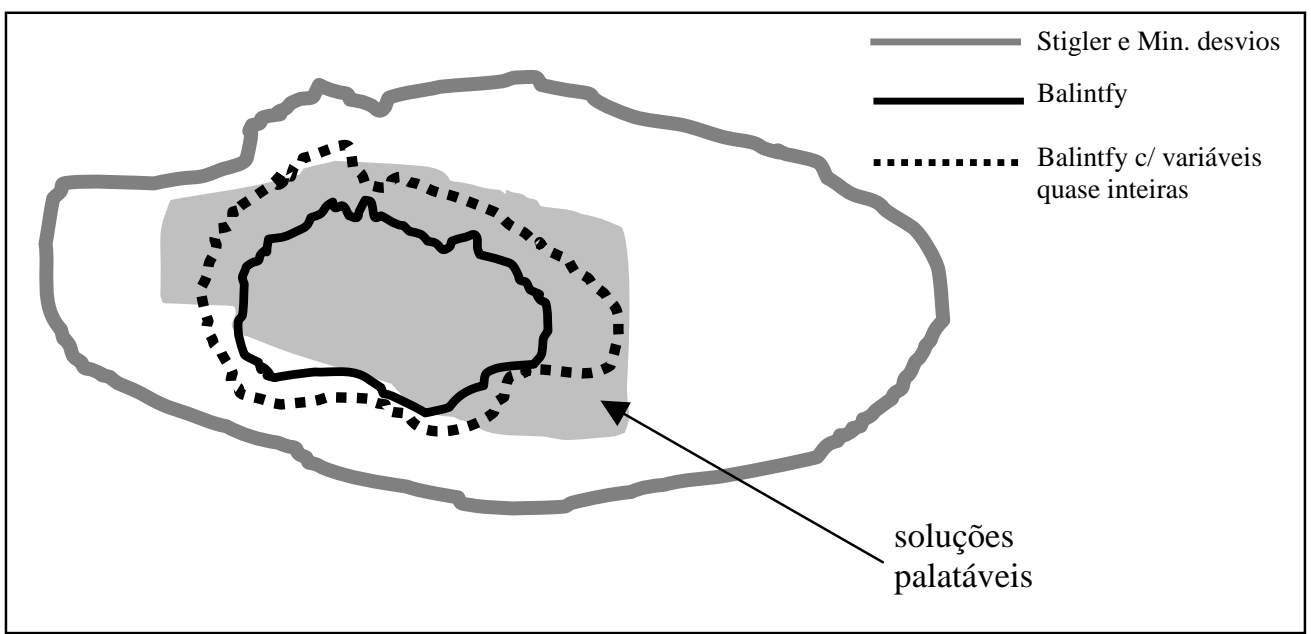

Figura 5 - Comparação entre os modelos matemáticos

Assumindo que as áreas limitadas pelas curvas indicam os espaços de soluções dos diferentes modelos e que a região hachurada indica o espaço de soluções palatáveis, podemos observar que quanto menor a liberdade de variação das quantidades de alimentos, menor o espaço de soluções viáveis e, consequentemente, pior a solução ótima. Desse modo, o modelo original de Stigler, por permitir liberdade total em relação às quantidades de alimentos, gera o menor custo mas também gera dietas com menor grau de palatabilidade. A partir da inclusão de restrições adicionais ao modelo de Stigler, a palatabilidade melhora, mas o espaço de soluções diminui e o custo aumenta. No outro extremo, o modelo de Balintfy utiliza quantidades fixas e pré-definidas de alimentos, implicando em um menor espaço de soluções e maior custo mas, em contrapartida, produzindo mais dietas viáveis para consumo. $\mathrm{O}$ modelo que utiliza variáveis quase-inteiras, por sua vez, amplia o espaço de soluções do modelo de Balintfy, pois permite pequenas variações nas quantidades pré-definidas de alimentos. Isso permite uma melhoria na função objetivo sem grandes impactos na palatabilidade da dieta. Para efeito de comparação através da Figura 5, consideramos o modelo de minimização de desvios sem a restrição de custo mínimo. Conforme pode ser verificado, neste caso, tanto o modelo de Stigler como o modelo de minimização de desvios apresentam o mesmo conjunto de soluções viáveis.

É importante ressaltar que foi fundamental a ordem em que foram rodados os modelos. O resultado inicial obtido com o modelo de Stigler possibilitou a introdução de restrições visando à melhoria da palatabilidade da dieta. Os resultados obtidos com a introdução das restrições possibilitaram a especificação das quantidades pré-definidas de alimentos utilizadas no modelo de Balintfy. Este último, por sua vez, deu origem ao modelo quaseinteiro.

Com relação ao modelo de minimização de desvios de nutrientes (Anderson \& Earle, 1983) poderíamos dizer que, apesar dos autores não mencionarem explicitamente a questão da palatabilidade, o modelo, aparentemente, deixa implícito uma certa ligação entre este aspecto e um balanceamento adequado de nutrientes. Por exemplo, a quantidade absurda de feijão $(268,49 \mathrm{~g})$ existente nos resultados do modelo de Stigler foi eliminada diminuindo-se os excedentes de alguns nutrientes (principalmente o ferro). O mesmo ocorreu com a couve e o 
óleo de soja e o resultado gerado para o modelo com minimização de desvios foi muito mais palatável que o modelo original de Stigler.

Em seu clássico livro Walden, publicado pela primeira vez em 1854 e fonte de inspiração do movimento hippie, Henry Thoreau (2001) afirma: "aprendi com a experiência de dois anos que custaria inacreditavelmente pouco trabalho obter-se a alimentação necessária ... e que o homem pode adotar uma dieta tão simples quanto os animais e ainda assim conservar-se saudável e forte". O que está por trás da afirmação de Thoreau é que é possível obter uma dieta simples (e, portanto, barata) e ao mesmo tempo saudável. Esta suposição está de certa forma implícita na maioria dos modelos de Programação Matemática apresentados nesse trabalho.

Além do custo e da saúde existe, no entanto, para o ser humano, a questão do paladar, questão esta de formulação bem mais complicada. Na natureza é feita a associação entre o prazer, ou seja, o gosto, e a necessidade, ou seja, aquilo que é necessário para tornar o animal ou vegetal saudável. Assim, na floresta, o animal ou vegetal procura prioritariamente os alimentos necessários à sua boa nutrição.

Em principio, o vínculo ainda existente entre o ser humano e a natureza, nos leva a admitir a validade, ao menos em parte, de idéias semelhantes. Embora isto não seja explicitado por Anderson \& Earle, poderia supor-se que é isto que está por trás de seu modelo. Ao minimizar os desvios em relação a um padrão alimentar, possivelmente Anderson \& Earle objetivavam atender melhor à questão da palatabilidade, questão essa totalmente ausente no modelo de Stigler.

Os fatos reais mostram, no entanto, que essas idéias não podem ser tomadas ao pé da letra. Basta verificar a difusão das cadeias de fast food e a popularidade de doces e frituras. Analisar o porque dessa situação vai além do escopo deste trabalho. Não pode haver, no entanto, sombra de dúvida que muitas distorções nos hábitos alimentares devem-se a efeitos de publicidade, padrões difundidos pelos meios de comunicação (o filme Super Size Me de Morgan Spurlock, EUA, 2004, sobre a cadeia McDonald's, ilustra bem estas idéias). Nada mais justo que a esta publicidade contraponham-se informações e esclarecimentos. Isto em parte já é feito nas escolas, em aulas de biologia ou ciências, campanhas de saúde pública veiculadas através da mídia ou feitas em hospitais, postos de saúde ou através de agentes de saúde. Além disso, a obrigação da embalagem do produto conter dados esclarecendo o valor nutricional do alimento representa um passo importante neste sentido. Acreditamos também que estudos como o presente, em que recebem destaque aspectos nutricionais da alimentação, significam uma contribuição dentro deste mesmo espírito.

A estes fatores, de natureza nutricional, a maioria dos modelos aqui apresentados agrega um elemento adicional, representando o lado econômico da questão. Que não necessariamente existe uma contradição absoluta entre o lado econômico e o lado nutricional, ou seja, dito de uma maneira simples, que não necessariamente o bom é caro e que tampouco, necessariamente, o caro é bom, esta é uma idéia já defendida parágrafos acima (veja Thoreau).

Evidentemente, na definição dos requisitos nutricionais dos modelos houve a participação de nutricionistas de forma implícita através dos dados e tabelas utilizadas. Cabe lembrar que estas informações, que definem níveis diários máximos e, principalmente, mínimos dos nutrientes necessários, envolvem sempre a participação destes especialistas. No trabalho aqui apresentado, além disso, foi consultada opinião de nutricionista com respeito a questões como palatabilidade, definição das porções dos alimentos, etc. Seria, no entanto, desejável uma interação mais intensa com o nutricionista, em que esse estivesse presente no terminal 
participando de forma direta na tomada de decisões, como, por exemplo, na introdução de restrições, redefinição do tamanho das porções, introdução de novos alimentos, modificação dos requisitos nutricionais, etc. Na verdade, a situação ideal seria que o software fosse operado diretamente pelo nutricionista, ficando o especialista de Pesquisa Operacional por conta de alguma dúvida ou esclarecimento técnico. Acreditamos que o software é suficientemente simples e amigável para permitir esta alternativa.

Finalmente, uma outra utilidade do software aqui apresentado, encontra-se na área didática, no ensino de técnicas de Pesquisa Operacional, formulação de modelos e algoritmos. Por tratar-se de um problema muito próximo da realidade - a alimentação - o software pode estimular e auxiliar os estudantes no processo de aprendizagem de modelagem e de uso de técnicas de Pesquisa Operacional. Através da interação com a ferramenta, permitindo a definição de diferentes parâmetros, a introdução de restrições adicionais e escolha dos diferentes modelos matemáticos, comparação e avaliação dos resultados obtidos, os estudantes podem enriquecer o seu conhecimento teórico e visualizar as aplicações da Programação Linear e Inteira aos problemas de nosso dia a dia. Visando possibilitar o uso desta ferramenta, estamos disponibilizando uma cópia do software SimulaDieta na secretaria da SOBRAPO.

\section{Referências Bibliográficas}

(1) Agricultural Research Service. (1975). The Thrifty Food Plan, U.S. Department of Agriculture, Hyattsville, Maryland.

(2) Anderson, A.M. \& Earle, M.D. (1983). Diet planning in the third world by linear and goal programming. Journal of the Operational Research Society, 34(1), 9-16.

(3) Armstrong, R.D. \& Sinha, P. (1974). Application of quasi-integer programming to the solution of menu planning problems with variable portion size. Management Science, 21(4), 480-488.

(4) Balintfy, J.L. (1975). A mathematical programming system for food management applications. Interfaces, 6, 13-31.

(5) Balintfy, J.L. (1979). The Cost of Decent Subsistence. Management Science, 25(10), 980-989.

(6) Balintfy, J.L.; Duffy, W.J. \& Sinha, P. (1974). Modeling Food Preferences Over Time. Operations Research, 22(4), 711-727.

(7) Balintfy, J.L.; Ross, G.T.; Sinha, P. \& Zoltners, A.A. (1978). A Mathematical Programming System for Preference and Compatibility Maximized Menu Planning and Scheduling. Mathematical Programming, 15, 63-76.

(8) Balintfy, J.L.; Rook, S.R. \& Taj, S. (1996). The Index of Decent Subsistence. Socio-Economic Planning Science, 30(4), 237-244.

(9) Bénichou, M.; Gauthier, J.M.; Girodet, P.; Hentges, O.; Ribiere, O. \& Vincent, O. (1971). Experiments in Mixed-Integer Linear Programming. Mathematical Programming, 1, 76-94.

(10) Bosch, R.A. (1993). Big Mac Attack. OR/MS Today, 20(4), 30-31. 
(11) Czyzyk, J.; Wisniewski, T. \& Wright, S.J. (1999). Optimization Case Studies in NEOS Guide. SIAM Review, 41(1), 148-163.

(12) Dantzig, G.B. (1963). Linear Programming and Extensions. Princeton University Press, Princeton, New Jersey.

(13) Dantzig, G.B. (1990). The Diet Problem. Interfaces, 20(4), 43-47.

(14) Erkut, E. (1994). Big Mac Attack Revisited. OR/MS Today, 21(3), 50-52.

(15) France, J. (1982). Using a Programmable Calculator for Rationing Beef Cattle. Journal of the Operational Research Society, 33, 419-429.

(16) Franco, G. (2003). Tabela de Composição Química dos Alimentos. 9ª. ed., Editora Atheneu, São Paulo.

(17) Garille, S.G. \& Gass, S.I. (2001). Stigler's Diet Problem Revisited. Operations Research, 49(1), 1-13.

(18) Gass, S.I. (1958). Linear Programming: Methods and Applications. McGraw-Hill Book Company, New York.

(19) Glen, J.J. (1980). A Parametric Programming Method for Beef Cattle Ration Formulation. Journal of the Operational Research Society, 31(8), 689-698.

(20) Glen, J.J. (1983). A Dynamic Programming Model for Pig Production. Journal of the Operational Research Society, 34(6), 511-519.

(21) IBGE (1996). Tabelas de Composição de Alimentos. $4^{\mathrm{a}}$. ed., IBGE, Rio de Janeiro.

(22) IBGE (2002). Tabelas de Preços de Alimentos - 2002. IBGE, Rio de Janeiro.

(23) Institute of Medicine - Food and Nutrition Board (2001). DRI - Diet Reference Intakes. National Academy Press, Washington, D.C.

(24) Kao, R.C. (1962). Linear Programming. Document number P-2514, RAND Corporation.

(25) Kraus, M.V. \& Mahan, L.K. (1985). Alimentos, nutrição e dietoterapia. 8a. ed., Ed. Roca, São Paulo.

(26) Lancaster, L.M. (1987). Algorithmic interface with computer graphics for a decision optimization system. Ph.D. Thesis, School of Management, University of Massachussets, Amherst, MA.

(27) Lancaster, L.M. (1992). The history of the application of mathematical programming to menu planning. European Journal of Operational Research, 57, 339-347.

(28) Lazarus, P. \& Kirkman, D. (1980). An Investigation of Feedstuffs for Dairy Cattle on Liverton Farm. Journal of the Operational Research Society, 31(1), 3-15.

(29) Liberatore, M.J.; \& Nydick, R.L. (1999). Breaking the Mold - A New Approach to Teaching the First MBA Course in Management Science. Interfaces, 29(4), 99-116.

(30) Lyons D.F. \& Dodd, V.A. (1976). The mix-feed Problem. Proceedings of the $7^{\text {th }}$ IFORS International Conference on Operational Research [edited by K.B. Halley]. 
(31) Lustig, I. (2001). Entrevista realizada com George Dantzig. Disponível em $<w w w . e-o p t m i z a t i o n . c o m>$.

(32) Pogrund, R.S. (1966). Nutrition in the Postattack Environment. Document number RM-5052-TAB, RAND Corporation.

(33) Schrage, L. (1997). Optimization Modeling with LINDO. Brooks/Cole Publishing Company, Pacific Grove, California.

(34) Smith, V.E. (1963). Electronic Computation of Human Diets. Michigan State University, Lansing, Michigan.

(35) Stigler, G. (1945). The Cost of Subsistence. Journal of Farm Economics, 25, 303-314.

(36) Thoreau, H.D. (2001). Walden. Editora Aquariana, São Paulo. 